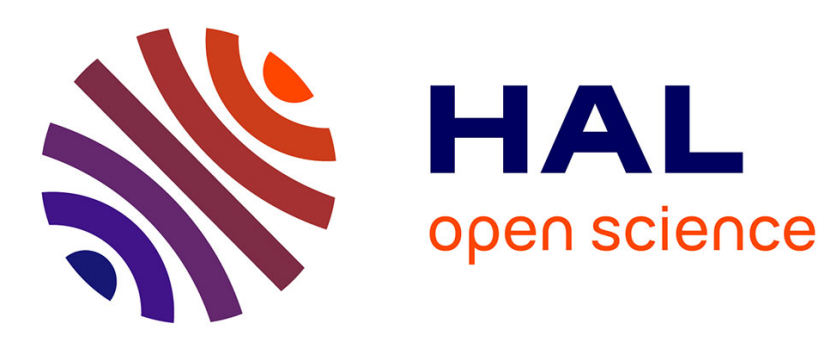

\title{
3D-Bottom Tracking based on Acoustic Diffraction for Autonomous Underwater Vehicles
}

Vincent Creuze, Bruno Jouvencel, Philippe Baccou

\section{To cite this version:}

Vincent Creuze, Bruno Jouvencel, Philippe Baccou. 3D-Bottom Tracking based on Acoustic Diffraction for Autonomous Underwater Vehicles. ICAR 2005 - 12th International Conference on Advanced Robotics, Jul 2005, Seattle, United States. lirmm-00106462

\section{HAL Id: lirmm-00106462 https://hal-lirmm.ccsd.cnrs.fr/lirmm-00106462}

Submitted on 16 Oct 2006

HAL is a multi-disciplinary open access archive for the deposit and dissemination of scientific research documents, whether they are published or not. The documents may come from teaching and research institutions in France or abroad, or from public or private research centers.
L'archive ouverte pluridisciplinaire HAL, est destinée au dépôt et à la diffusion de documents scientifiques de niveau recherche, publiés ou non, émanant des établissements d'enseignement et de recherche français ou étrangers, des laboratoires publics ou privés. 


\title{
3D-Bottom Tracking based on Acoustic Diffraction for Autonomous Underwater Vehicles
}

\author{
V. Creuze, B. Jouvencel, P. Baccou \\ Laboratoire d'Informatique, de Robotique et de Microélectronique de Montpellier \\ (LIRMM / CNRS, Université Montpellier II - UMR 5506) \\ 161 rue Ada, 34392 Montpellier CX 5, France \\ research.creuze@laposte.net, jouvencel@lirmm.fr,philippe.baccou@wanadoo.fr
}

\begin{abstract}
Navigation of autonomous underwater vehicles (A.U.V.) in very shallow waters implies acoustic detection. In single beam sonar systems, sound emitted by ultrasonic transducers is diffracted and secondary lobes appear. Considering the sea bottom's backscattering properties, secondary lobes can be used to work out the three-dimensional equation of the plane that represents the seabed. In this paper, we will first consider characteristics of electro-acoustic transducers with rectangular aperture and study the resulting acoustic diffraction. Then, we explain how to choose the best dimensions of the transducer and the related best orientation. Moreover, we introduce a method aiming to extract the seabed $3 D$ equation from the received acoustic echo. Thus single beam sonar systems can be used for bottom tracking purposes. We present the results of our simulations and our experimental device.
\end{abstract}

Index Terms - Acoustic detection, acoustic diffraction, bottom tracking, Autonomous Underwater Vehicles.

\section{INTRODUCTION}

For some years, Autonomous Underwater Vehicles (A.U.V.) have been substantially developed. Due to industrial and scientific requirements, the necessity of navigating in very shallow water has emerged. And then, features like positioning [1][2], obstacle avoidance [3][4], bottom tracking [5], and target characteristics [6] have been studied.

Acoustic detection of the environment is achieved through multi-beam forward-looking sonar, lateral sonar, and now synthetic and interferometric sonar systems [7].

Many of these tasks require sophisticated equipment whose size, weight, and electrical consumption can be damaging to the vehicle's autonomy and its live load. This point is particularly critical for very small AUVs, like TAIPAN our laboratory's prototype.

For bottom tracking applications such as survey or video imaging another approach can be studied. In fact, with single beam acoustic emitters, the emitted acoustic wave is diffracted. Features of this diffracted beam can be computed by using emitter dimensions, sound speed in water, and signal frequency.

Moreover, combining diffraction of the emitted signal with backscattering properties of the bottom allows us to work out data that is traditionally not obtained with single beam acoustic systems.

In this paper, we present a method of threedimensional seabed detection based on the use of a single electro-acoustic transducer (sounder). This method uses both acoustic diffraction and bottom backscattering coefficients.

First, we present the acoustic sensor and explain how its rectangular aperture will diffract the acoustic beam. We also detail the bottom backscattering properties.

In the second part, we demonstrate that the choice of the best dimensions of the transducer is related to its angle of orientation. Then, we give the dimensions and orientation adapted to our purpose.

In the third part, we assume that the seabed is locally a plane and we propose a method allowing us to treat the acoustic echo received by the sensor. This method aims to work out the 3D equation of the seabed plane.

The last part consists in the presentation of our simulations and our experimental device.

\section{SOUND DIFFRACTION AND BACKSCATTERING}

\section{A. Assumptions}

We consider an autonomous underwater vehicle fitted with a single electro acoustic transducer (Fig.1) in its front part (Fig.3 and Fig.10). The vehicle keeps its pitch angle always null, i.e. the vehicle remains horizontal along its displacements. The angle of orientation between transducer axis and the horizontal axis $(O x)$ is called $\psi$ (Fig.1). The emitted signal is a sine pulse whose frequency is $f=200 \mathrm{kHz}$. The transmit pulse length is called $\tau$.

We also assume that the seabed consists of rocks and that it is sufficiently regular to be considered locally as a plane.

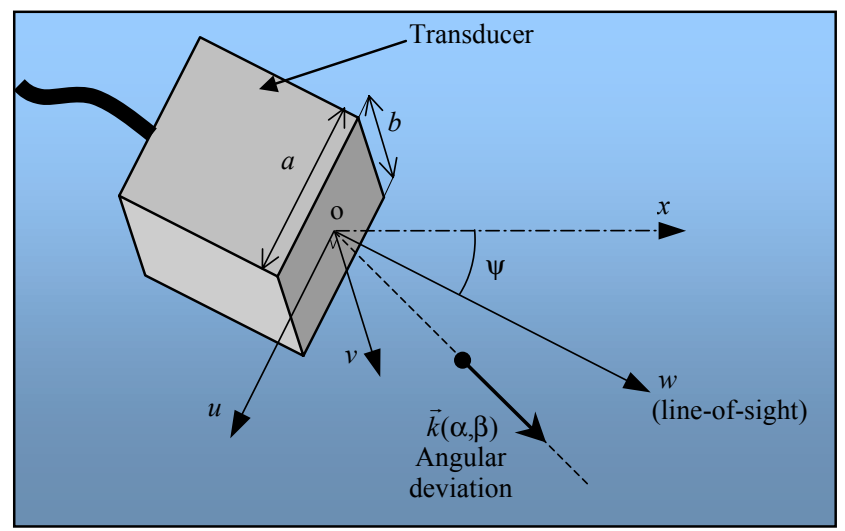

Fig. 1. Schematic view of the transducer 


\section{B. Sound diffraction}

When dimensions of the aperture of the transducer are not very large $(a$ or $b<20 \mathrm{~mm})$ in comparison with the wavelength $(\lambda=7.5 \mathrm{~mm}$ at $f=200 \mathrm{kHz}$ ), diffraction must be taken into account in the estimation of beam geometry. For distances $R$ greater than $\lambda / r_{c}$ (Fraunhofer zone) one can determine the beam geometry by application of the Huygens-Fresnel principle.

Sound intensity $I(R, \alpha, \beta)$ at range $R$ and angular deviation $(\alpha, \beta)$ from the line-of-sight of the transducer (Fig. 1) is given by:

$$
I(R, \theta)=I_{o}(R)\left(\frac{\sin p}{p}\right)^{2}\left(\frac{\sin q}{q}\right)^{2}
$$

with $p=\frac{\pi a \alpha}{\lambda}$ and $q=\frac{\pi b \beta}{\lambda}$

where $\lambda$ is the sound wavelength, $a$ and $b$ are respectively the length and the width of the aperture of the transducer, $\alpha$ and $\beta$ are the coordinates of the vector of angular deviation (Fig. 1), and $I_{o}(R)$ is the sound intensity at range $R$ on the beam axis [8]. $I_{o}(R)$ depends on transmission loss and will be detailed in the next paragraph.

The well-known diffraction pattern thus obtained shows a main lobe and some secondary lobes, which intensity decays very quickly (Fig. 2). The angles between the axis of the main lobe (also called the line-of-sight of the transducer) and the axes of each of the two secondary lobes along $\boldsymbol{u}$ and $\boldsymbol{v}$ directions (Fig. 1) are called respectively $\theta_{\mathrm{a}}$ and $\theta_{\mathrm{b}}$. They are given by:

$$
\theta_{\mathrm{a}}=\arcsin \left(\frac{3 \lambda}{2 a}\right) \quad \text { and } \quad \theta_{\mathrm{b}}=\arcsin \left(\frac{3 \lambda}{2 b}\right)
$$

where $\lambda$ is the sound wavelength, $a$ and $b$ are respectively the length and the width of the aperture of the transducer.

Due to the backscattering properties of the seabed (see next section), it is most of time sufficient to take into account only the main lobe and the two inferior secondary lobes (Fig. 3).

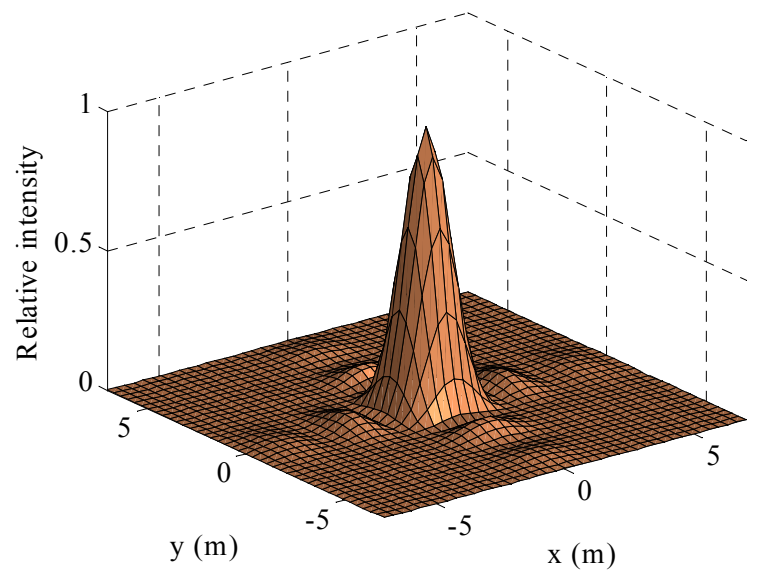

Fig. 2. Diffraction pattern obtained with a rectangular aperture

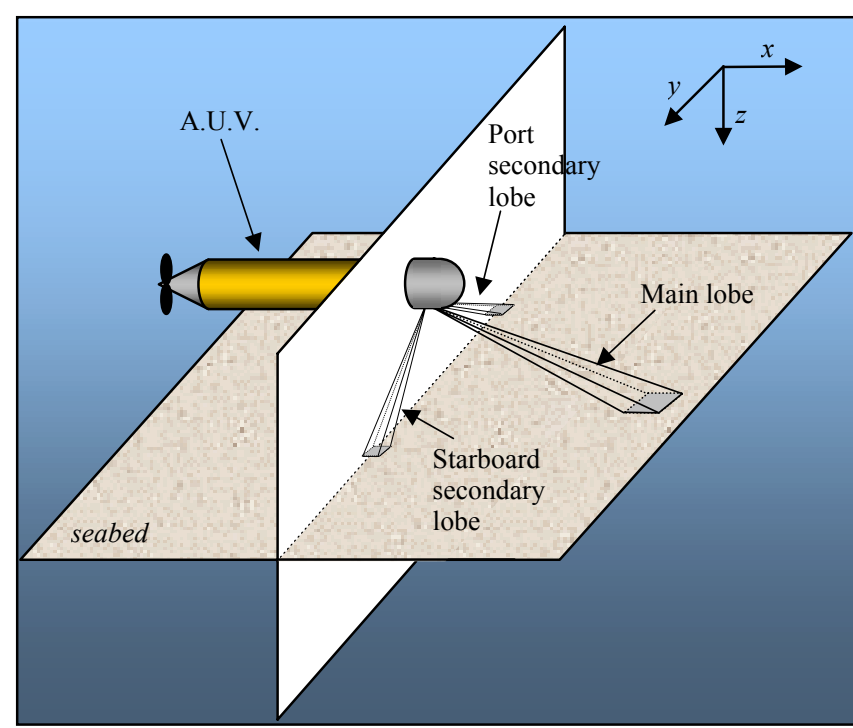

Fig. 3. The AUV and three lobes of the diffracted acoustic beam

Angular attenuation of intensity (in $\mathrm{dB}$ ) is called $\operatorname{diffrac}_{d B}(\alpha, \beta)$ and is given by:

$$
\operatorname{diffrac}_{d B}(\alpha, \beta)=10 \log \left(\frac{\sin p}{p}\right)^{2}\left(\frac{\sin q}{q}\right)^{2}
$$

\section{Transmission loss}

The transmission loss $(T L)$ consists of two parts, one due to spherical spreading, the other due to absorption loss in the water:

$$
T L=10 \log R^{2}+\alpha \cdot 10^{-3} \cdot R
$$

where $R$ is the range in meters, and $\alpha$ the absorption coefficient (in $\mathrm{dB} / \mathrm{km}$ ) and is related to the square of frequency [9].

\section{Backscattering}

Once it has reached the seabed, the acoustic wave will be partially backscattered. The backscattering coefficient, $B S$, varies with the incidence angle $\varphi$ (Fig.4) and is given in $\mathrm{dB} / \mathrm{m}^{2}$. So, backscattered intensity will depend both on incidence angle but also on the extent of the seabed area that contributes to the backscattered signal.

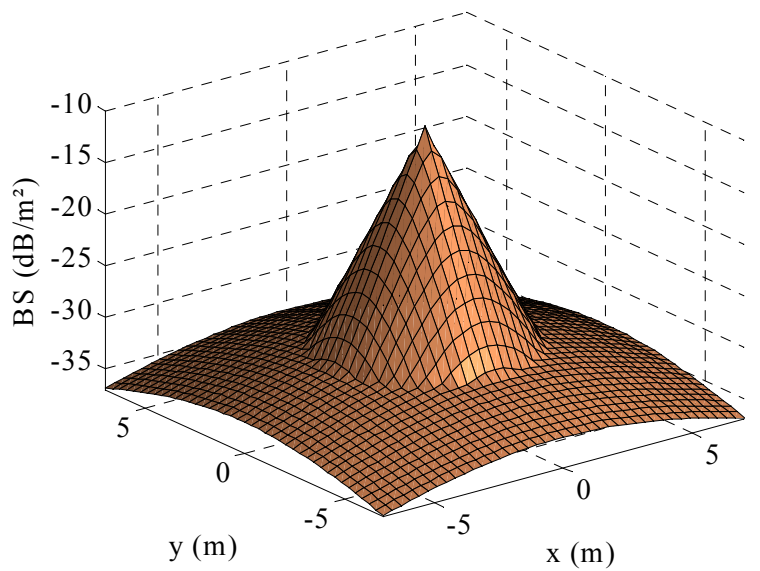

Fig. 4. Backscattering coefficient (in dB) 
For incidence angles greater than about $25^{\circ}$ a good approximation of backscattering coefficient $B S$ is given by Lambert's law [10]:

$$
B S=B S_{o}+20 \log \left(\cos \varphi_{\mathrm{i}}\right)
$$

where $B S_{o}$ is a constant coefficient depending on seabed characteristics, and $\varphi_{\mathrm{i}}$ is the incidence angle.

For incidence angles smaller than $25^{\circ}$ one can approximate backscattering coefficient BS by:

$$
B S=B S_{N}+\frac{\varphi_{i}}{\varphi_{o}}\left(B S_{o}-B S_{N}+20 \log \left(\cos \varphi_{o}\right)\right)
$$

where $B S_{N}$ is the backscattering coefficient for normal incidence, $B S_{o}$ is the backscattering coefficient used in Lambert's law (5), $\varphi_{\mathrm{o}}$ is the transition angle $\left(=25^{\circ}\right)$, and $\varphi_{\mathrm{i}}$ is the incidence angle.

In order to take into account the extent of the seabed area that contributes to the backscattered signal, $B S$ has to be normalized. The normalized backscattering coefficient is called BTS and is given by:

$$
B T S=B S+10 \log (\sigma)
$$

where $\sigma$ represents the extent of the seabed area which contributes to the backscattered signal and depends on beam geometry and transmit pulse length.

\section{E. Total attenuation}

In order to calculate sound intensity of the signal backscattered by each seabed part, we determine $H$, the total attenuation per unit area (8) by adding $\mathrm{dB}$ values of attenuation due to diffraction (3), two-way transmission loss (4), and backscattering (7).

$$
H=\operatorname{diffrac}_{d B}-2 T L+B T S
$$

\section{FEATURING THE TRANSDUCER}

\section{A. Relationship between dimensions and orientation}

Considering (5) and (6), one can say that an acoustic echo will be all the more backscattered, as the angle of incidence will be smaller. This is especially crucial for secondary lobes, because they do not carry much acoustic energy. Thus, secondary lobes must be placed in the vertical plane $(O y z)$ in order to ensure best backscattering conditions.

Let us call $\mathbf{A}, \mathbf{B}$, and $\mathbf{C}$ the unitary vectors representing respectively the axes of the right (starboard) and left (port) secondary lobes and the axis of the main lobe. To simplify the problem, we assume that the aperture of the transducer is square $(a=b)$. Considering (2), one can say that $\theta_{\mathrm{a}}=\theta_{\mathrm{b}}$ and we introduce $\theta=\theta_{\mathrm{a}}=\theta_{\mathrm{b}}$. Then the angle between the axis of the main lobe and the plane can be computed as follows:

In the $(O, \boldsymbol{u}, \boldsymbol{v}, \boldsymbol{w})$ frame (Fig.1), vectors $\mathbf{A}$ and $\mathbf{B}$ are written $\mathbf{A}=\left(\mathrm{A}_{u}, \mathrm{~A}_{v}, \mathrm{~A}_{\mathrm{w}}\right)$ and $\mathbf{B}=\left(\mathrm{B}_{u}, \mathrm{~B}_{v}, \mathrm{~B}_{w}\right)$
Due to the theory of diffraction, we have:

$$
\left\{\begin{array}{l}
\mathrm{A}_{v}=0 \\
\mathrm{~A}_{w}=\mathrm{A} \sin \left(\frac{\pi}{2}-\theta_{a}\right) \\
\mathrm{B}_{u}=0 \\
\mathrm{~B}_{w}=\mathrm{B} \sin \left(\frac{\pi}{2}-\theta_{b}\right)
\end{array}\right.
$$
we have:

As $\mathbf{A}$ and $\mathbf{B}$ are unitary vectors and $\theta=\theta_{\mathrm{a}}=\theta_{\mathrm{b}}$,

$$
\text { A.B }=\mathrm{A}_{u} \mathrm{~B}_{u}+\mathrm{A}_{v} \mathrm{~B}_{v}+\mathrm{A}_{w} \mathrm{~B}_{w}=\sin ^{2}\left(\frac{\pi}{2}-\theta\right)
$$

Let us call $\xi$ the angle between $\mathbf{C}$ and the vertical plane defined by $\mathbf{A}$ and $\mathbf{B}$. Due to symmetry reasons and because $\mathbf{C}$ is an unitary vector, we have:

$$
\mathbf{C} .(\mathbf{A}+\mathbf{B})=\|\mathbf{C}\| .\|\mathbf{A}+\mathbf{B}\| \cdot \cos (\xi)=\|\mathbf{A}+\mathbf{B}\| \cdot \cos (\xi)
$$

Considering that $(\mathbf{A}+\mathbf{B}) \cdot(\mathbf{A}+\mathbf{B})=\|\mathbf{A}+\mathbf{B}\|^{2}$, we have: $\|\mathbf{A}+\mathbf{B}\|=\sqrt{2+2 \mathbf{A} \cdot \mathbf{B}}$. Because of (10), we have:

$$
\|\mathbf{A}+\mathbf{B}\|=\sqrt{2+2 \sin ^{2}\left(\frac{\pi}{2}-\theta\right)}
$$

By definition, we have also A.C $=\mathbf{B} \cdot \mathbf{C}=\cos (\theta)$. As $\mathbf{C} .(\mathbf{A}+\mathbf{B})=\mathbf{C . A}+\mathbf{C . B}$, we can write:

$$
\text { C. }(\mathbf{A}+\mathbf{B})=2 \cos (\theta)
$$

Combining (11), (12) and (13) gives us:

$$
\cos \xi=\frac{2 \cos (\theta)}{\sqrt{2+2 \sin ^{2}\left(\frac{\pi}{2}-\theta\right)}}
$$

Finally, $\psi$ the best angle of orientation of the transducer should allow secondary lobes to be in a vertical plane. The angle $\psi$ corresponds to the one between the line-of-sight of the transducer (i.e. the axis of the main lobe) and the horizontal plane. Replacing $\theta$ by (2) in (14), we obtain (15) where $\psi$ depends only on $a$ the dimension of aperture of the transducer (Fig. 5).

$$
\psi=\frac{\pi}{2}-\arccos \frac{2 \cos \left(\arcsin \frac{3 \lambda}{2 a}\right)}{\sqrt{2+2 \sin ^{2}\left(\frac{\pi}{2}-\arcsin \frac{3 \lambda}{2 a}\right)}}
$$

\section{B. Choice of the dimensions}

Once $\mathbf{A}$ and $\mathbf{B}$ are in a vertical plane, the value of their angle of incidence on a flat bottom has to be chosen the smallest as possible (in order to enhance backscattering). Considering the symmetry of the problem and using (10) and (2), this angle of incidence, called $i$, is easily obtained by:

$$
i=\frac{1}{2} \operatorname{arcos}(\mathbf{A} \cdot \mathbf{B})=\frac{1}{2} \operatorname{arcos}\left(\sin ^{2}\left(\frac{\pi}{2}-\arcsin \frac{3 \lambda}{2 a}\right)\right)
$$

For the choice of the dimensions of the aperture, we must however take into account $\rho$, the ratio between the distances covered by main and secondary beams. The lowest value of $\rho$ is 1 , but this ratio $\rho$ has to be maximized so that the echoes of main and secondary lobes do not occur simultaneously and then can be easier experimentally detected. 
The ratio $\rho$ to be maximized is defined as:

$$
\rho=\frac{\frac{\text { depth }}{\sin \psi}}{\frac{\text { depth }}{\cos i}}=\frac{\cos i}{\sin \psi}
$$

where $i$ and $\psi$ are defined by (16) and (15).

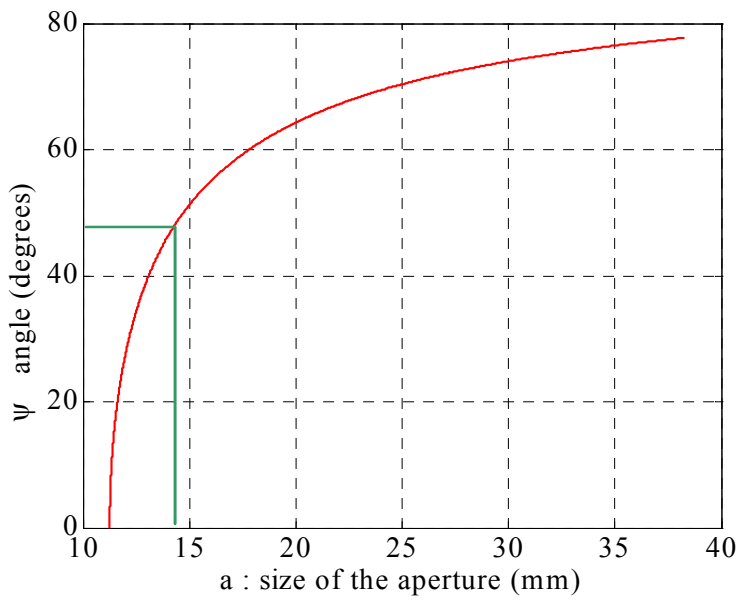

Fig. 5. Best angle of orientation depending on the aperture

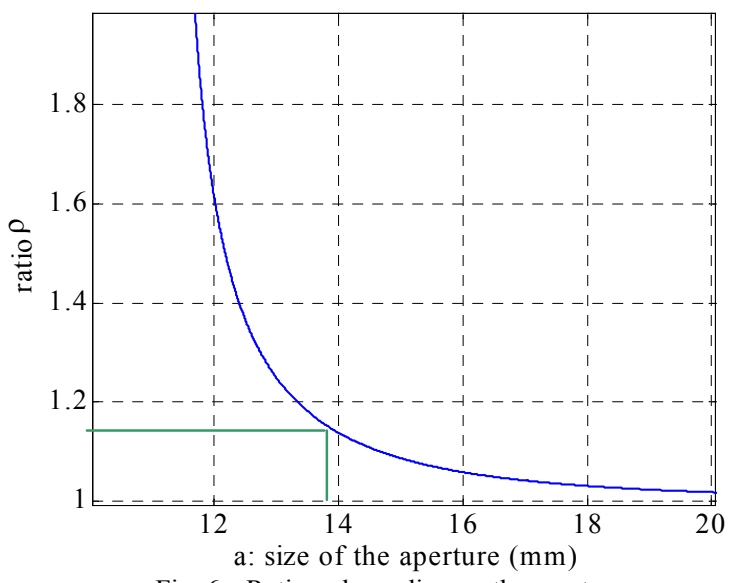

Fig. 6a. Ratio $\rho$ depending on the aperture

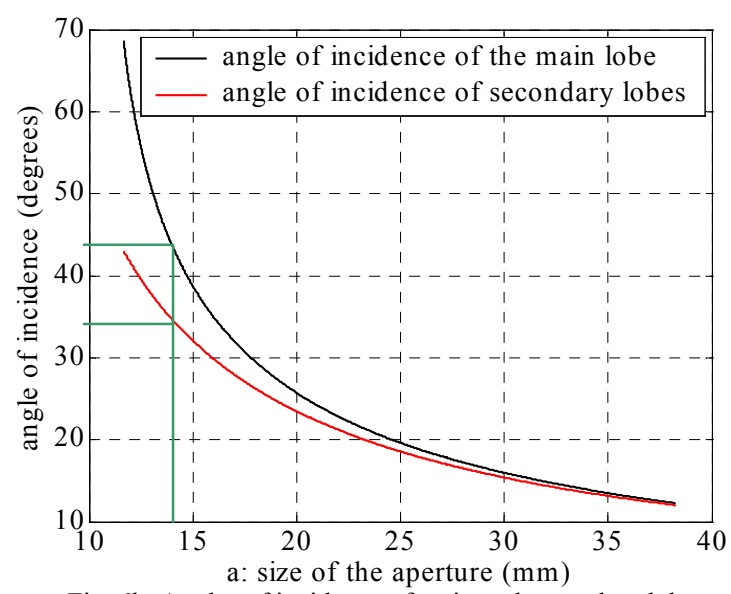

Fig. 6b. Angles of incidence of main and secondary lobes

Finally, the choice of the dimensions of the aperture of the transducer has to be made so as to maximize $\rho$ (Fig. 6a) and $\psi$ (Fig. 5). Moreover, one must not only minimize $i$ the angle of incidence of secondary lobes, but also maximize the angle of incidence of the main lobe (Fig. 6b), so as to ensure balanced backscattered acoustic levels for main and secondary lobes.

On the previous graphs one can see that $a=13.9 \mathrm{~mm}$ is a good compromise for the size of the aperture of the transducer. Then, $\psi$ the corresponding angle of orientation of the transducer is given by (15) and we have $\psi=45.7^{\circ}$. The obtained value $\rho=1.18$ ensures a good ability to distinguish main and secondary echoes. These echoes are well balanced due to incidence angles respectively set to $45^{\circ}$ and $32^{\circ}$.

The value $a=13.9 \mathrm{~mm}$ will be the reference value in the next sections of this paper.

\section{SEABED EQUATION}

\section{A. Received sound pressure}

Considering $\S \mathrm{II}$, sound pressure $s(t)$ of the backscattered signal is given by:

$$
s(t)=\iint_{S} e(t-\Delta t(x, y)) 10^{\frac{H(x, y)}{20}} d x d y
$$

where $e(t)$ is the sound pressure pulse emitted by the transducer, $S$ is the extent of the seabed area which contributes to the echo, $(x, y)$ are coordinates on seabed, $\Delta t(x, y)$ is the delay defined by:

$$
\Delta t(x, y)=2 \frac{R(x, y)}{c}
$$

where $R(x, y)$ is the distance between the transducer and the coordinates $(x, y)$ on seabed, and $c$ is the sound speed in the water, and $H(x, y)$ is the attenuation (in $\mathrm{dB}$ ):

$$
H(x, y)=\operatorname{diffrac}_{d B}(x, y)-2 \operatorname{TL}(x, y)+B T S(x, y)
$$

Due to the angles of incidence of main and secondary lobes (Fig.6), backscattering level BTS is lower for main lobe signal than for secondary lobes. The same effect occurs for transmission loss $T L$. Consequently, in spite of Fraunhofer attenuation, global attenuation level $H$ makes secondary lobes echo level significant in comparison with main lobe echo level.

Thus, the echo received by the transducer is made of one big peak corresponding to the echo of the main lobe and one or two smaller peaks corresponding to echoes of secondary lobes (Fig.7). It has to be noticed that there are some cases where these peaks cannot be distinguished because they are superimposed (see next section).

It also has to be noticed that maximums of pressure peaks correspond to axes of main and secondary lobes only when angular decrease due to the diffraction attenuation is larger than angular decrease of backscattering coefficients. The validity of this assumption depends on $B S_{o}$ and $B S_{N}$ backscattering coefficients but is verified in most of cases. 


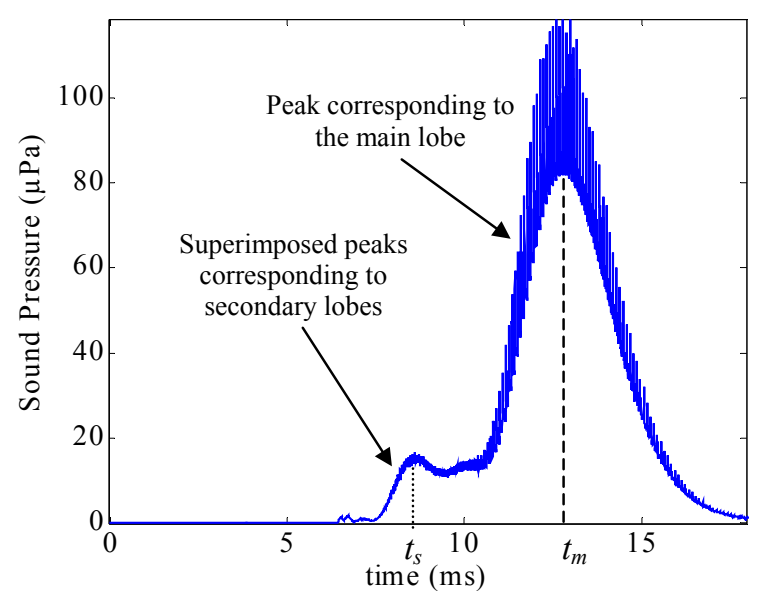

Fig. 7. An example of acoustic echo with two peaks

\section{B. Three-dimensional equation of the seabed}

The temporal evolution of the sound pressure of the backscattered signal allows us to work out the threedimensional equation of the bottom, which is locally assumed to be the equation of a plane (21).

$$
\alpha . x+\beta . y+\gamma \cdot z+\delta=0
$$

where $\alpha, \beta$, and $\delta$ are constants depending on the seabed slope and depth, and $\gamma$ is conventionally set to $\gamma=1$.

The $(\mathrm{O}, \mathrm{x}, \mathrm{y}, \mathrm{z})$ frame is centered on the transducer and oriented as shown on (Fig. 3). Then, $\delta$ is directly obtained as the opposite of the vertical distance between the transducer and the seabed. Constants $\alpha$ and $\beta$ are related respectively to the slopes along $(\mathrm{Ox})$ and $(\mathrm{Oy})$ direction.

Three cases may occur:

\section{- Two Peaks (Fig.7):}

Echoes due to secondary lobes are superimposed. Then, one can say that the slope along $(O y)$ direction is null and we have immediately $\beta=0$.

$t_{s}$ denotes the instant of occurrence of the maximum of the first peak and $t_{m}$ the instant of occurrence of the maximum of the main peak (Fig. 7). Then, simple geometrical considerations give us:

$$
\left.\begin{array}{l}
\delta=-\frac{c}{2} t_{s} \cos i \\
\beta=0 \\
\alpha=\frac{-\delta}{\frac{c}{2} t_{m} \cos \psi}-\tan \psi
\end{array}\right\}
$$

where $c$ is the sound speed, and $i$ and $\psi$ are given by (16) and (15).

\section{- $\quad$ More than two peaks (Fig.8):}

Echoes due to secondary lobes are not superimposed and echoes due to tertiary lobes may also occur.

$t_{s 1}$ and $t_{s 2}$ denote the instants of occurrence of the maximum of the two highest first peaks and $t_{m}$ the instant of occurrence of the main peak maximum (Fig. 8). Then we have:

$$
\left.\begin{array}{l}
\delta=-\frac{c}{4}\left(\mathrm{t}_{\mathrm{s} 1}+\mathrm{t}_{\mathrm{s} 2}\right) \\
\beta= \pm \frac{2 \delta-c t_{s 1} \cos i}{c t_{s 1} \sin i} \\
\alpha=\frac{-\delta}{\frac{c}{2} t_{m} \cos \psi}-\tan \psi
\end{array}\right\}
$$

where $c$ is the sound speed, and $i$ and $\psi$ are given by (16) and (15). The uncertainty of sign of $\beta$ is not discussed in this paper but can be solved by several means: using previous values, briefly modifying the roll angle of the vehicle, using a rectangular aperture for the transducer...

\section{- $\quad$ Only one Peak (Fig.9):}

Echoes due to main and secondary lobes are superimposed. Then, it is impossible to determine $\beta$. However, one can approximate $\delta$ as to be:

$$
\delta \approx-\frac{c}{2} t_{\min }
$$

where $c$ is the sound speed, and $t_{\min }$ corresponds to the beginning of the peak (Fig.9).

The constant $\alpha$ cannot be computed but one can say that the slope along $(O y)$ direction is greater than a certain value. This value depends both on the beam width of the main lobe and on the angle $\theta$ defined by (2). Thus, we have:

$$
\alpha>\tan \left(\frac{\theta}{2}-\frac{1}{2} \arcsin \frac{\lambda}{a}\right)
$$

\section{SIMULATIONS AND EXPERIMENTAL DEVICE}

\section{A. Simulations}

Simulations have been conducted under Matlab ${ }^{\mathrm{TM}}$ environment. The seabed is represented by a grid whose mesh size is ten times smaller than $c \times \tau$, where $c$ is the sound speed and $\tau$ is the transmit pulse length. given by:

The sound pressure $p_{\text {echo }}(t)$ of the received echo is

$$
p_{\text {echo }}(t)=\sum_{i j} p_{\text {emitted }}\left(t-\Delta t_{i j}\right) \cdot 10^{\frac{H_{i j}}{20}}
$$

where $p_{\text {emitted }}$ is the emitted acoustic pressure at 1 meter along the line-of-sight of the transducer, $(i, j)$ are coordinates in the seabed grid, $H_{i j}$ is the attenuation (in $\mathrm{dB})$, and $\Delta t$ is given by (19).

In the following example, we have considered a transducer whose aperture is a 13.9 millimeters wide square. The values of backscattering coefficients are: $B S_{o}$ $=-35 \mathrm{~dB}$ and $B S_{N}=-10 \mathrm{~dB}$. The transition angle of Lambert's law is $\varphi_{0}=20^{\circ}$. The angle of orientation of the transducer is $\psi=45^{\circ}$ and the vehicle is supposed to navigate deep enough to neglect the effects of the surface on sound propagation and echo.

Parameters of the seabed equation used in the presented simulations are the following $(\alpha=-0.1, \beta=0$, $\gamma=1, \delta=-5.5)$ for Fig. $7,(\alpha=0, \beta=0.56, \gamma=1, \delta=-5.5)$ for Fig. 8 , and $(\alpha=0.11, \beta=0, \gamma=1, \delta=-8)$ for Fig. 9 . 


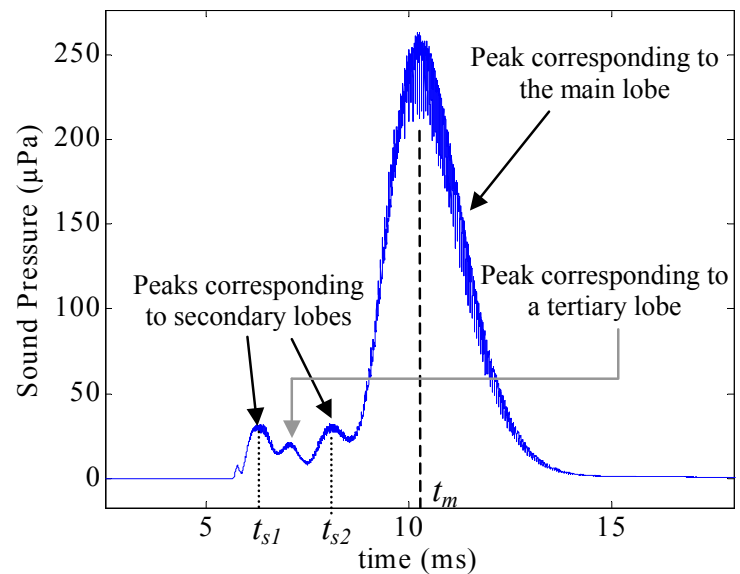

Fig. 8. Simulation of an acoustic echo with more than two peaks

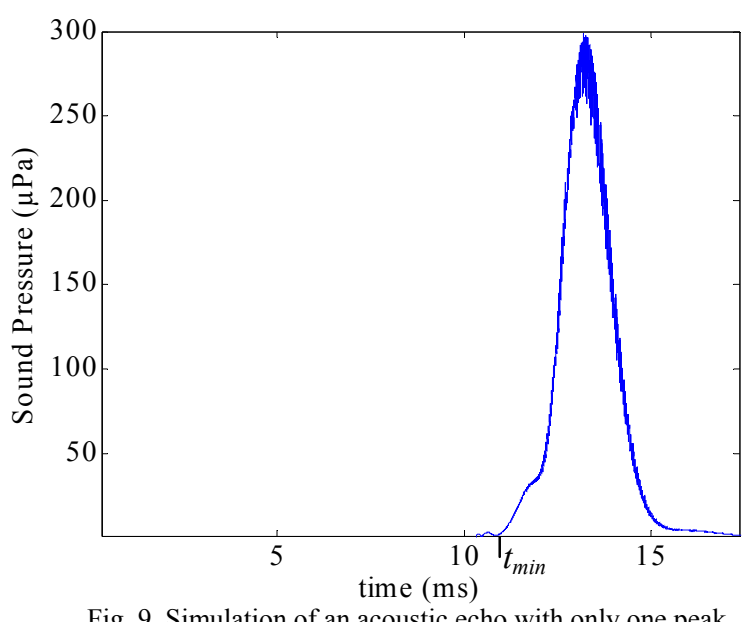

Fig. 9. Simulation of an acoustic echo with only one peak

The simulations have been conducted with many orientations of the bottom and each time the comparison with the obtained seabed equation has confirmed the liability of the presented method.

For value of $\beta$ greater than 0.57 , we have noticed the occurrence of echoes of tertiary or quaternary lobes that avoid the method to work properly and can conduct to aberrant results.

Finally, simulations show that the presented method is valid until $-0.9<\alpha<0.11$ and $-0.57<\beta<0.57$. This means that the seabed slope along $(\mathrm{Ox})$ axis must be superior to $-42^{\circ}$ and inferior to $7^{\circ}$ (this value could be improved by increasing $a$ ), and seabed slope along (Oy) axes must remain between $\pm 29^{\circ}$.

\section{B. Experimental device}

In order to perform experimental validation, we will use an electro-acoustic transducer whose operating frequency is $200 \mathrm{kHz}$. A specific electronic circuit has already been designed. It is computer controlled and one can choose the transmit pulse length. A Time Varying Gain is applied to the backscattered signal so that it is at an optimum level for the 12 bits A/D converter. Then, the received signal is sent to the computer and processed.

This device has already been tested and will be soon implemented on our laboratory's new A.U.V. prototype "Taipan II" (Fig. 10) in order to perform bottom tracking.
This paper describes how Fraunhofer diffraction can be used as an advantage in detection of the seabed equation. We have demonstrated what should be the best dimension and orientation of the transducer in order to achieve bottom tracking. We have also explained how to treat the received acoustic echo to determine the threedimensional equation of the bottom (that is locally supposed to be a plane). Simulations have been conducted and their results should be soon validated by experimentation in the open sea. The designed device will be implemented on our new small AUV prototype "Taipan II" (Fig. 10). Presently, our research concerns how to combine the obtained acoustic echoes with the DVZ concept [11] to achieve bottom tracking.

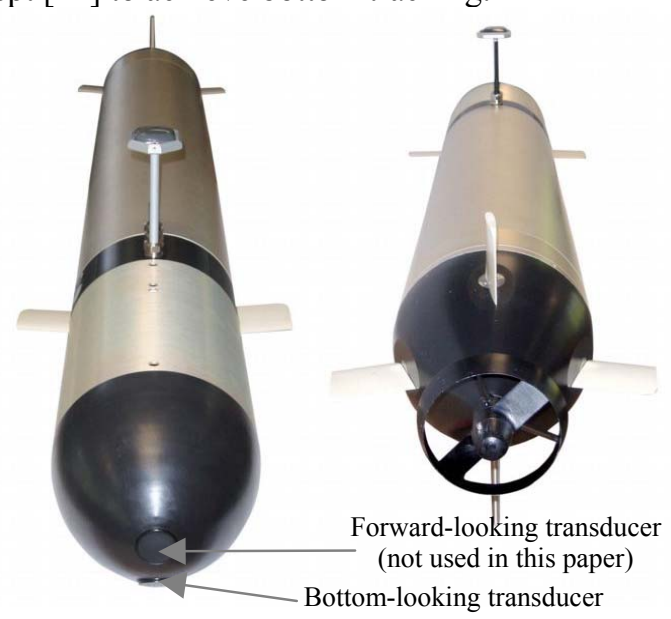

Fig. 10. Taipan II and its electro acoustic transducers

\section{REFERENCES}

[1] J.J. Leonard and H.F. Durrant-Whyte, "Directed Sonar Sensing for Mobile Robot Navigation," ser. The Kluwer International Series in Engineering and Computer Science, Norwell, MA: Kluwer, 1992.

[2] P. Baccou, B. Jouvencel, and V. Creuze, "Homing and Navigation Using One Transponder," IEEE ICRA2002, Washington D.C., May 2002.

[3] Y. Petillot, I. Tena Ruiz, and D. M. Lane, "Underwater Vehicle Obstacle Avoidance Using a Multi-Beam Forward Looking Sonar," IEEE Journal of Oceanic Engineering, Vol. 26, no. 2, pp. 240-251, April 2001.

[4] V. Creuze and B. Jouvencel, "Avoidance of Underwater Cliffs for Autonomous Underwater Vehicles," IEEE IROS 2002, Lausanne, Switzerland, pp. 793-798, October 2002.

[5] V.Creuze and B. Jouvencel, "Perception et suivi de fond pour véhicules autonomes sous-marins", Traitement du Signal, 2003 Vol. 20 no. 4, pp. 323-336, December 2003.

[6] J.R. Edwards, H. Schmidt, and K.D. LePage, "Bistatic Synthetic Aperture Target Detection and Imaging with an AUV," IEEE Journal of Oceanic Engineering, Vol. 26, no. 4, pp. 690-699, October 2001.

[7] Y. Perrot, B. Hamonic, and M. Legris, “Three dimensional imaging using SAS interferometry", Underwater Acoustics ECUA 2000, Vol. 1, pp. 425-431, July 2000.

[8] A.D. Pierce, Acoustics, an introduction to its physical principles and applications, McGraw Hill, New-York, 1981.

[9] Robert J. Urick, Principles of Underwater Sound, Peninsula Publishing, 1983.

[10] X. Lurton, An introduction to Underwater Acoustics - Principles and Applications, Springer Praxis Books, 2003.

[11] R. Zapata, P. Lépinay, "Collision avoidance and bottom following of a torpedo-like vehicle," MTS/IEEE OCEANS96, Fort Lauderdale, Florida, September 1996. 